\title{
Flexible High-Conductivity Carbon-Nanotube Interconnects Made by Rolling and Printing
}

\author{
S. Tawfick, K. O’Brien, and A. J. Hart*
}

\begin{abstract}
$\boldsymbol{A}_{p}$ pplications of carbon nanotubes (CNTs) in flexible and complementary metal-oxide-semiconductor (CMOS)-based electronic and energy devices are impeded due to typically low CNT areal densities, growth temperatures that are incompatible with device substrates, and challenges in large-area alignment and interconnection. A scalable method for continuous fabrication and transfer printing of dense horizontally aligned CNT (HA-CNT) ribbon interconnects is presented. The process combines vertically aligned CNT (VA-CNT) growth by thermal chemical vapor deposition, a novel mechanical rolling process to transform the VA-CNTs to HA-CNTs, and adhesion-controlled transfer printing without needing a carrier film. The rolling force determines the HA-CNT packing fraction and the HA-CNTs are processed by conventional lithography. An electrical resistivity of $2 \mathrm{~m} \Omega \cdot \mathrm{cm}$ is measured for ribbons having 800-nm thickness, while the resistivity of copper is 100 times lower, a value that exceeds most CNT assemblies made to date, and significant improvements can be made in CNT structural quality. This rolling and printing process could be scaled to full wafer areas and more complex architectures such as continuous CNT sheets and multidirectional patterns could be achieved by straightforward design of the CNT growth process and/or multiple rolling and printing sequences.
\end{abstract}

\author{
Keywords: \\ - alignment \\ - carbon nanotubes \\ - interconnects \\ - rolling \\ - transfer printing
}

\section{Introduction}

Owing to the outstanding properties ${ }^{[1,2]}$ of individual carbon nanotubes (CNTs), thin films of densely packed horizontally aligned CNTs (HA-CNTs) could find applications as electrical, mechanical, thermal, and multifunctional structures in microdevices. In particular, theoretical and experi-

\footnotetext{
[*] Prof. A. J. Hart, S. Tawfick

Department of Mechanical Engineering

University of Michigan

2350 Hayward St., Ann Arbor, MI 48109 (USA)

E-mail: ajohnh@umich.edu

K. O’Brien

Intel Corportation

2501 NW 229th Ave

RA3 - 252, Hillsboro, OR 97214 (USA)

马upporting Information is available on the WWW under http:// www.small-journal.com or from the author.
}

DOI: $10.1002 /$ smll.200900741 mental studies confirm that CNTs are resistant to electromigration at emerging complementary metal-oxidesemiconductor (CMOS) linewidths, ${ }^{[3]}$ have micrometer-scale electron mean free paths, ${ }^{[4]}$ and have a higher current carrying capacity than $\mathrm{Cu}\left(10^{9} \mathrm{~A} \mathrm{~cm}^{-2}\right) \cdot{ }^{[5]}$ Therefore, highly organized CNTs may eventually replace $\mathrm{Cu}$ in both vertical and horizontal microelectronic interconnects. ${ }^{[6 a]}$ Owing to the outstanding bending resilience of CNTs and the insensitivity of their electrical resistance to mechanical deformation, ${ }^{[6 \mathrm{~b}]}$ highconductivity films are ideal for use in flexible and stretchable electronics.

Three major challenges ${ }^{[3]}$ must be met in scaling the electrical properties of CNTs into assemblies, and to thereby consider CNTs as a manufacturable interconnect technology: i) metallic CNTs must be densely packed in the desired vertical or horizontal orientation; ii) processing conditions including CNT growth must be compatible with CMOS processing over wafer-scale areas; and iii) low-resistance ohmic contact must be made to all graphene shells (walls) of the CNTs. Further, as key performance parameters (e.g., mean free path, number of 


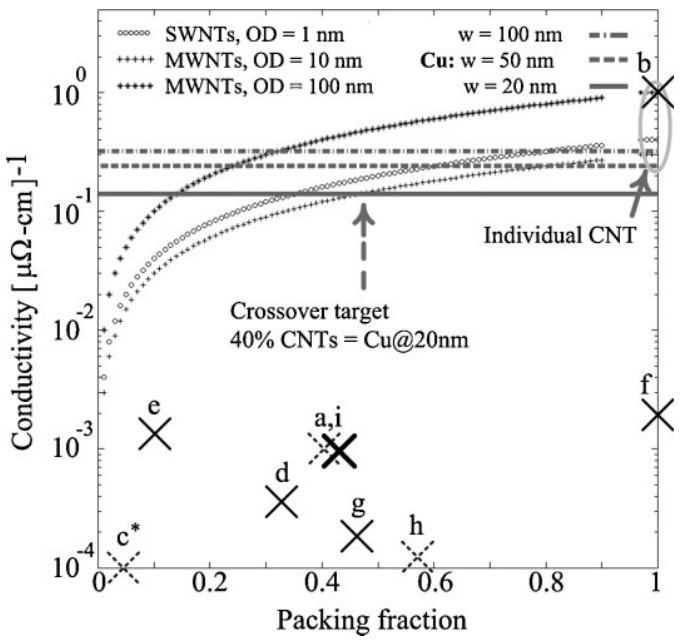

Figure 1. Previously published calculations of electrical conductivity along continuous CNTs as related to the CNT packing fraction, compared to previously measured electrical data for individual CNTs and CNT networks. The packing fraction is defined as the as the ratio of the CNT areal density (number of CNTs per $\mathrm{cm}^{2}$ ) to the areal density of hexagonally packed CNTs (modeled as cylinders) having the same outer diameter. Solid and dashed X marks are MWCNT and SWCNT, respectively. We assume that the conductivity of an aligned CNT network is linearly proportional to the number of CNTs per unit cross-sectional area and that the constant of proportionality is the conductivity of an individual CNT. ${ }^{[4,25]}$ Data point (i) is for the HA-CNT ribbons described in this study.

conduction channels) depend on CNT length and diameter, an ideal manufacturing process would facilitate tuning of CNT diameter as well as build interconnects from continuous parallel CNTs. Growth of vertically aligned CNT (VA-CNT) "forests" $[7]$ is an effective means of orienting large numbers of CNTs having a monodisperse diameter distribution defined by the catalyst nanoparticles, however, the packing density of high-quality VA-CNTs is typically $1 \%-2 \%$ and no more than $10 \%,{ }^{[8-10]}$ which is insufficient for bulk applications. Notable previous electrical measurements of individual CNTs and CNT assemblies are summarized in Figure 1 and Table 1. While theoretical performance has been matched by measurements of invididual CNTs, the measured electrical conductivity values of $\mathrm{CNT}$ assemblies to date are typically at least two orders of magnitude lower than theory predicts for transport through continuous aligned CNTs at the same packing fraction. Electrical performance is strongly dependent on the structural characteristics and contact conditions of the CNT assembly. Manufacturing of densely packed HA-CNT assemblies having high electrical conductivity would be a major step forward toward applications of CNTs in thin film and flexible devices.

We create thin ribbons of HA-CNTs on arbitrary substrates by mechanical transformation of VA-CNT forests grown on a silicon substrate followed by printing using a poly(dimethysiloxane) (PDMS) carrier substrate. Prior approaches to HACNT fabrication include growth aligned by crystallographic interactions with the substrate,${ }^{[11]}$ growth aligned by a gas flow over the substrate ${ }^{[12]}$ and by dielectrophoresis of CNTs in solution. ${ }^{[13]}$ However, HA-CNT growth is limited to very low density and multilayer approaches such as repeated transfer printing of sparse arrangements, ${ }^{[14]}$ which require an impractical number of cycles. Capillary forces have been used to transform VA-CNTs into HA-CNTs such as by controlled dipping of VA-CNT microstructures into a liquid such as isopropanol or acetone.$^{[8,15]}$ In this alternative process, the final CNT density is a result of two competing forces: the force that draws CNTs together as the solvent infiltrates the CNT assembly then evaporates, and the elastic forces associated with deforming the CNTs. Therefore, the final packing density is limited by the initial spacing between the CNTs. ${ }^{[16]}$ Further, we find that capillary forces deform high-aspect-ratio VA-CNT structures, and therefore cannot address millimeter-long CNTs as discussed herein.

\section{Results and Discussion}

VA-CNT microstructures are first grown by thermal chemical vapor deposition (CVD) on a silicon wafer, then the VA-CNTs are transformed into densely packed HA-CNTs by direct mechanical contact using a small roller (Figure 2a). A supported catalyst thin film $\left(1 / 10 \mathrm{~nm} \mathrm{Fe} / \mathrm{Al}_{2} \mathrm{O}_{3}\right)$ is deposited by electron-beam evaporation and this film is lithographically patterned on a silicon wafer coated with 500-nm thermallygrown $\mathrm{SiO}_{2}$. The VA-CNT microstructures resembling thin "blades" are grown to $1-2 \mathrm{~mm}$ height by atmospheric pressure CVD in $\mathrm{C}_{2} \mathrm{H}_{4} / \mathrm{H}_{2} / \mathrm{He}$, at $775^{\circ} \mathrm{C}^{[17]} \mathrm{As} \mathrm{reported} \mathrm{by} \mathrm{Pint} \mathrm{et} \mathrm{al.,}{ }^{[18]}$ strong adhesion between the VA-CNT blades and the substrate is created by rapid cooling in the reaction atmosphere, so as to

Table 1. Details of CNT electrical data plotted in Figure 1, cited from previous studies of individual CNTs and CNT assemblies.

\begin{tabular}{llcccc}
\hline Label in Figure 1 & CNT type & Diameter & Packing fraction & Resistivity [m $\Omega \cdot \mathrm{cm}]$ & Reference \\
\hline $\mathrm{a}$ & SWCNT & $1.3 \mathrm{~nm}$ & 0.4 & 0.91 & $10^{-6}$ \\
b & MWCNT & $100 \mathrm{~nm}$ & individual & 33 & {$[26]$} \\
c $^{[\mathrm{a}]}$ & SWCNT & $1 \mathrm{~nm}$ & 0.01 & 2.5 & {$[4]$} \\
$\mathrm{d}$ & MWCNT & $12 \mathrm{~nm}$ & 0.33 & 0.7 & {$[11]$} \\
$\mathrm{e}$ & MWCNT & $7 \mathrm{~nm}$ & 0.10 & $5 \times 10^{-4}$ & {$[27]$} \\
$\mathrm{f}$ & MWCNT & $70 \mathrm{~nm}$ & individual & 5 & {$[29]$} \\
$\mathrm{g}$ & MWCNT & $15 \mathrm{~nm}$ & 0.45 & 8 & {$[19]$} \\
$\mathrm{h}$ & SWCNT & $2.8 \mathrm{~nm}$ & 0.58 & 1 & {$[15]$} \\
i & MWCNT & $10 \mathrm{~nm}$ & 0.42 & {$[\mathrm{~b}]$} \\
\hline
\end{tabular}

[a] Values of packing and resistivity were scaled linearly to fit within the axis limits of Figure 1. [b] Average of measurements taken on bundles fabricated by the method presented in this paper. 
a) VA-CNT blade $\approx 2 \%$ packing fraction

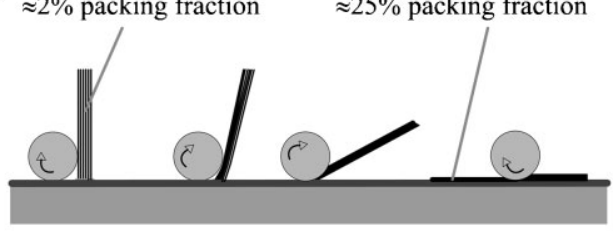

b)

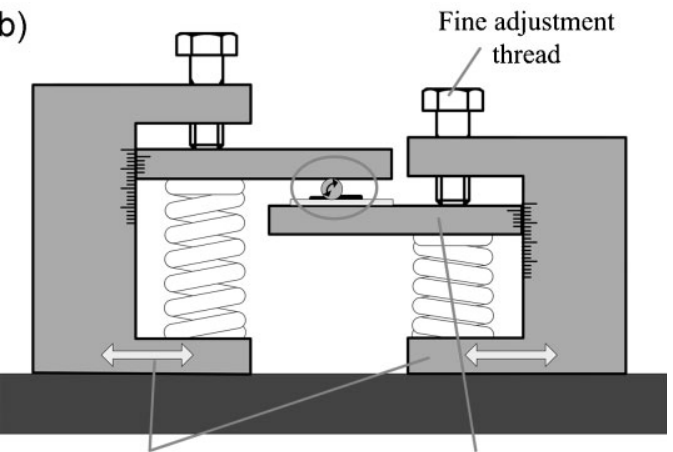

Translating axes

Spring-loaded platform (rolling force control)

C) Support rails

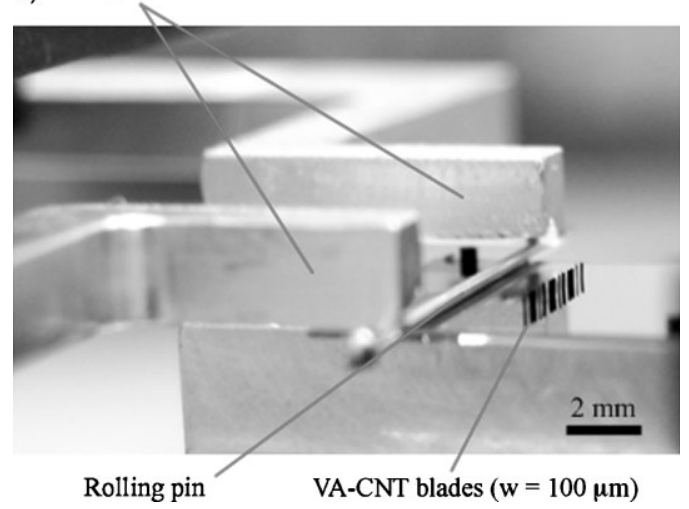

Figure 2. Rolling of VA-CNT blades into densely packed HA-CNTs: a) kinematics of the rolling process; b) schematic of the force-controlled rolling machine; and c) optical image showing VA-CNT blades mounted in the machine before rolling.

form a carbide interface between the CNTs and catalyst particles. Alternatively, when He flow is used during the cooling step, VA-CNTs are easily delaminated from the substrate. Strong adhesion is useful when the CNTs remain on the growth substrate, whereas weak adhesion is useful when the CNTs are printed to a secondary substrate as described later.

After growth, the substrate with VA-CNT blades is placed in a custom-built benchtop apparatus (Figure 2b and c and Supporting Information, Figure S1), wherein a stainless steel roller is held between the substrate and a pair of rails. The substrate and rails are moved in opposite directions, causing the roller to rotate about a fixed virtual pivot. Contact between the roller and the CNTs first topples the VA-CNT blades to the horizontal orientation, and then the contact pressure between the roller and CNTs compacts the CNTs as the roller moves across the substrate. The applied force determines the thickness of the HA-CNT ribbons after rolling. The force is adjusted using springs in the machine frame.
Initial contact between a VA-CNT blade and the roller topples the blade by kinking the structure at its base, causing little shear in the upper portion of the blade. Minimizing shear and buckling of the VA-CNTs during the rolling process is essential for creating HA-CNT films with uniform texture, and this is achieved using a roller diameter less than the VA-CNT blade height and designing the VA-CNT blade width to be substantially less than the height. In the experiments reported here, we use a 0.6-mm-diameter stainless-steel pin to roll VACNTs with 1-2-mm initial height and 20-100- $\mu \mathrm{m}$ initial width. Our method contrasts recent approaches that shear a uniform VA-CNT forest into a thin film using a much larger diameter roller that is separated from the CNTs by a flexible mesh. ${ }^{[18,19]}$ In our experience with this alternative approach, entanglement and van der Waals forces among the VA-CNTs induce significant shear, and therefore the resulting CNT films are not well aligned through their thickness.

Further, for effective transformation, the HA-CNTs must not adhere to the roller. Since the resultant force from the van der Waals attraction between the CNTs and roller is directly proportional to the contact area, it is desirable to minimize the local contact area between the CNTs and the roller. From Hertzian contact mechanics, ${ }^{[20]}$ the width of the contact area between a cylinder (diameter $d$, length $l$, modulus $E_{1}$, Poisson's ratio $\left.v_{1}\right)$ and a plane $\left(E_{2}, v_{2}\right)$, is

$b=\left[\frac{2 F d}{\pi l}\left(\frac{1-v_{1}^{2}}{E_{1}}+\frac{1-v_{2}^{2}}{E_{2}}\right)\right]^{1 / 2}$

where $F$ is the normal rolling force.

Therefore, a small diameter roller made of a material having high elastic modulus gives relatively weak adhesion to the CNTs. This simple formula agrees with our observation that the CNTs tend to stick to rollers having a substantially larger

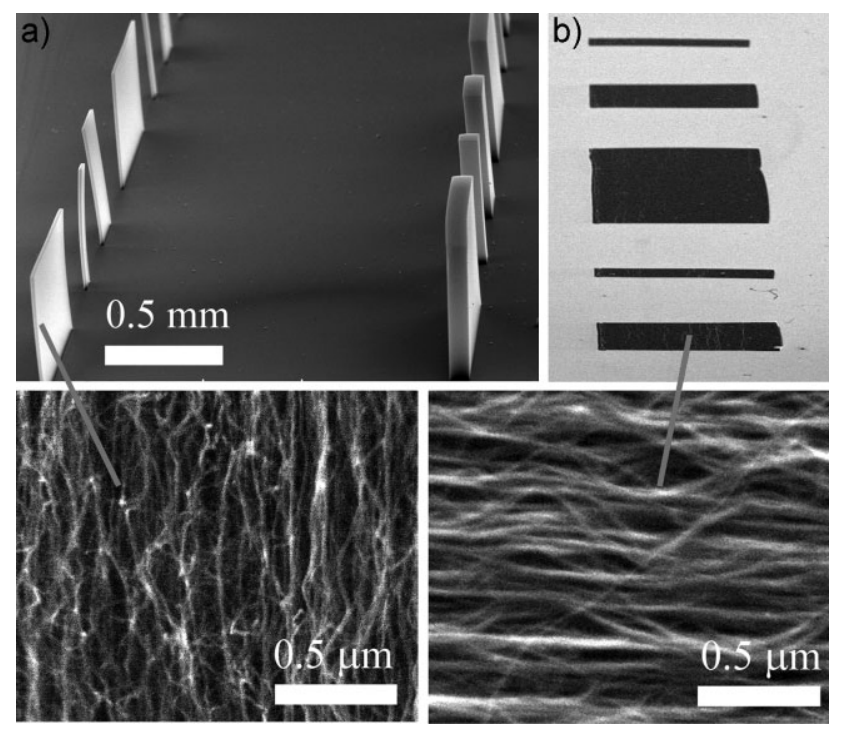

Figure 3. SEM images of a) as-grown VA-CNTs having 20 and $100 \mu \mathrm{m}$ width and b) HA-CNTs fabricated by rolling followed by capillary densification. Higher-magnification images show CNT alignment in each configuration. 
a)

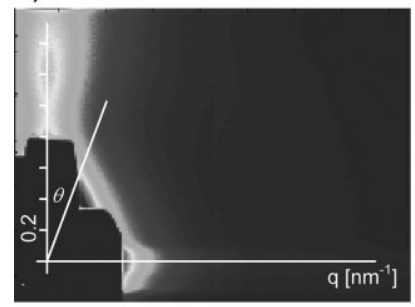

b)

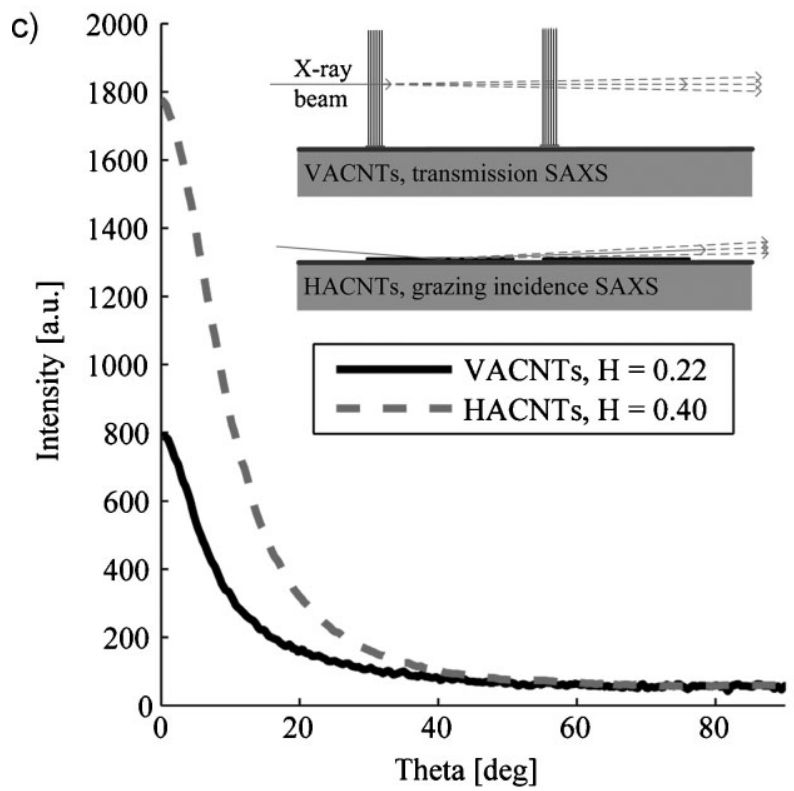

Figure 4. Characterization of CNT microstructures by synchrotron X-ray scattering showing aligned morphology: a) transmission SAXS image of VA-CNTs before rolling; $b$ ) grazing incidence SAXS image of HA-CNTs after rolling; and c) comparison of azimuthal intensity distributions.

diameter than used in the present study, and/or to rollers made of a soft material (e.g., PDMS or Nylon). Further, large rollers cause undesirable shearing and buckling of the VA-CNTs. (Figure S2)

Our force-controlled rolling process creates HA-CNT ribbons with uniform thickness and significantly higher packing density than the original VA-CNT blades (Figure 3). The rolling process preserves and possibly increases the CNT alignment, as quantified by calculating Herman's orientation parameter $(H)^{[21]}$ from synchrotron small-angle X-ray scattering (SAXS) data. Example SAXS images are shown in Figure 4. For VA-CNT blades characterized by transmission SAXS, $H=0.2$; for HA-CNT ribbons characterized by grazing incidence (GI-) SAXS, $H=0.4$. Precise comparison of $H$ values between the transmission and grazing incidence configurations is not possible as Herman's parameter is highly sensitive to background scattering. However, from the full-width-halfmaximum (FWHM) of the azimuthal intensity distribution, we can judge that the alignment of the HA-CNTs is at least as good as the initial VA-CNTs, and logically should increase as the CNTs are densified during processing. Further, as we expect that the X-ray beam penetrates fully through the HA-CNT ribbon thickness, SAXS verifies that the ribbons exhibit strong alignment through their thickness.
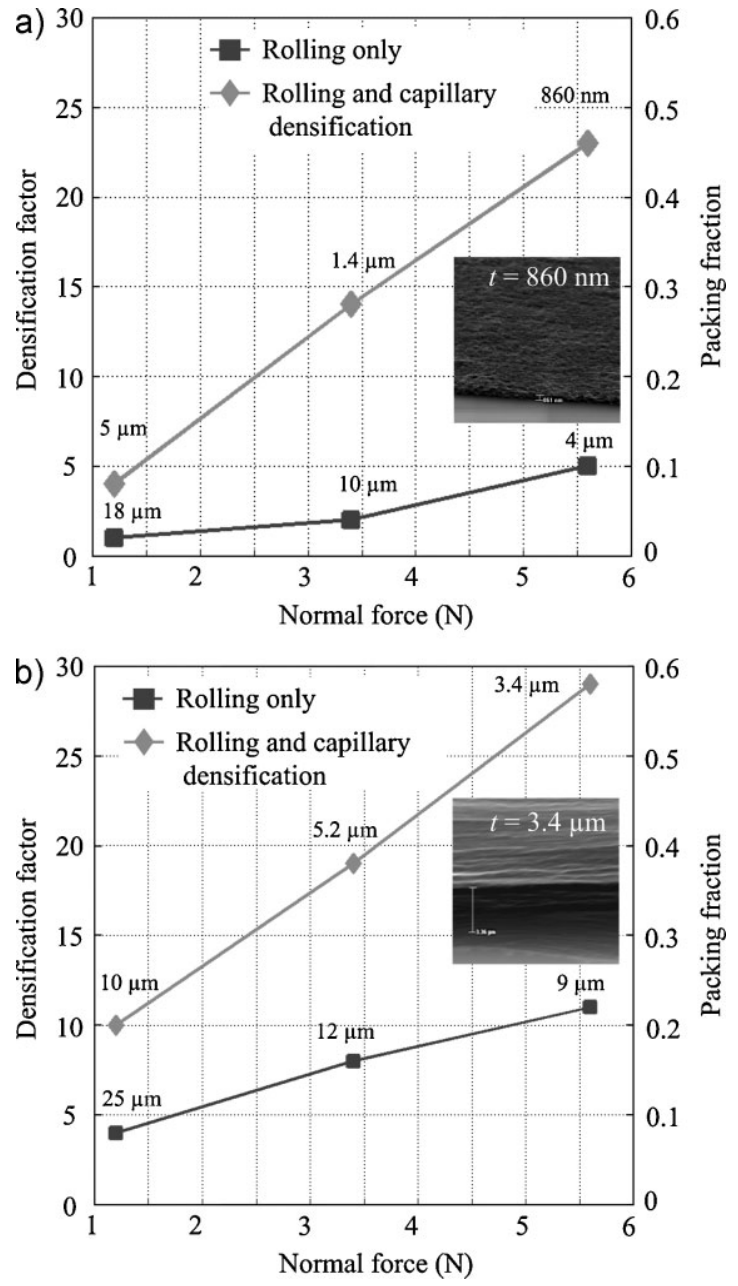

Figure 5. Control of HA-CNT geometry by control of rolling force: densification factor and packing fraction for a) $20-\mu \mathrm{m}$ and b) $100-\mu \mathrm{m}$ VACNT blade widths. Insets show side views of typical HA-CNT ribbons after rolling and capillary densification at $5.6 \mathrm{~N}$ applied force. Thickness values are written next to each data point and are averages for three samples processed under identical conditions.

The final thickness $(t)$ and packing density of the HA-CNT ribbons is controlled by the initial width $(w)$ of the VA-CNT blades prior to transformation, and by the force applied to the roller. Figure 5 plots the densification ratio $(w / t)$ and packing fraction of HA-CNT ribbons created from VA-CNT blades having $w=20 \mu \mathrm{m}$ and $100 \mu \mathrm{m}$. The densification factor increases linearly with the normal force applied during the rolling process. This linear plastic behavior is due to the initially low density of VA-CNTs; the CNTs are easily compacted in the direction perpendicular to their alignment. The HA-CNT density is calculated using the known VA-CNT density and the measured densification ratio. The VA-CNT density is calculated from measurements of the mass (using a microbalance) and the CNT microstructure volume (using scanning electron microscopy (SEM)) of a VA-CNT forest grown fully over a silicon substrate. The CNTs used in this study have an average outer diameter of $10 \mathrm{~nm}$ and an inner diameter of $6 \mathrm{~nm}$, as also determined by SAXS. ${ }^{[22]}$ The VA-CNT density is $0.02 \mathrm{~g} \mathrm{~cm}^{-3}$, corresponding to a packing fraction of 0.016 . The density at the 
maximum packing fraction of 0.9 is $1.26 \mathrm{~g} \mathrm{~cm}^{-3}$, and for comparison the density of graphite is $2.2 \mathrm{~g} \mathrm{~cm}^{-3}$.

After the rolling process, significant further densification is achieved by infiltrating the HA-CNTs with an organic solvent such as acetone and subsequently evaporating the solvent. Evaporation of the solvent draws the CNTs into a more tightly packed arrangement. In agreement with previous studies of elastocapillary aggregation of fibers and hair, ${ }^{[16]}$ the final packing fraction depends on the initial spacing (packing fraction) of the HA-CNTs after the rolling process. Therefore, maximal packing of HA-CNTs is achieved by combining mechanical and capillary densification methods. The maximum densification factor achieved in this study is 30 , giving a final packing fraction of approximately 0.6. Raman spectroscopy (Figure S3) shows that the G/D peak intensity ratio $\left(I_{\mathrm{G}} / I_{\mathrm{D}}\right)$ is invariant before and after rolling and densification, therefore suggesting that the CNTs are not damaged during the rolling and densification steps.

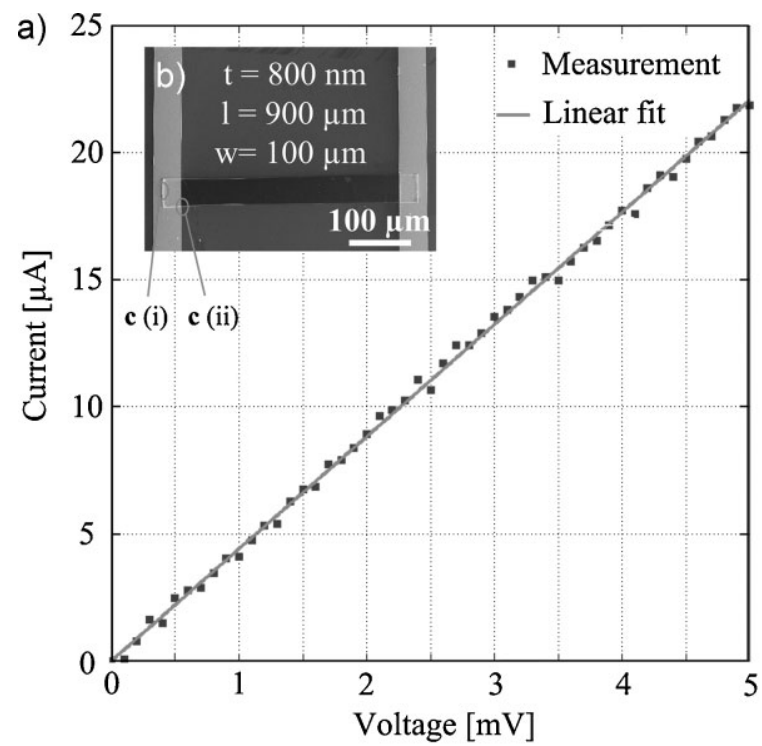

c) (i)

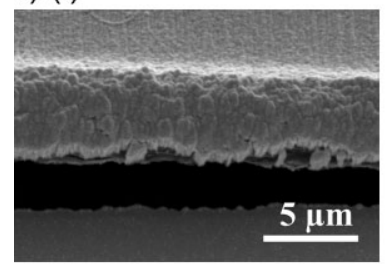

d)

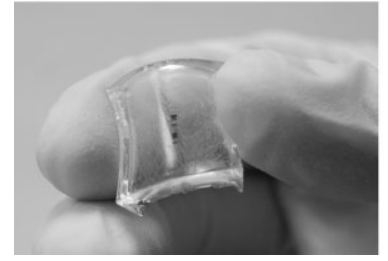

c) (ii)

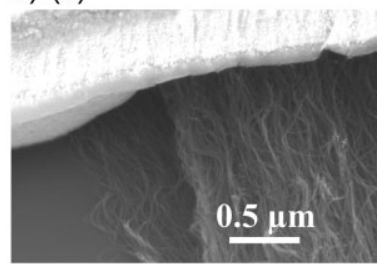

e)

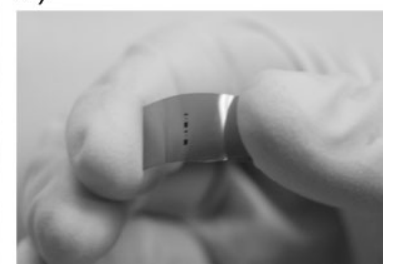

Figure 6. Electrical characterization of HA-CNT ribbons with packing fraction of $\approx 0.6$ and transfer printing to flexible substrates: a) linear $I-V$ curve; b) microfabricated HA-CNT film interconnect; c) views of Au-CNT contact, as labeled in (b); d) row of HA-CNT ribbons on PDMS; e) row of HACNT ribbons on Kapton film.
The HA-CNT ribbons can be processed by standard photolithography and plasma-etching methods, enabling straightforward device fabrication and electrical characterization. To make electrical contact to the ribbons and create prototype thin-film interconnects, the ribbons were masked by photolithography and their ends were exposed and etched in $\mathrm{O}_{2}$ plasma. Then, Au was deposited by electron-beam evaporation and patterned by lift-off (Figure 6a). Ribbons with thickness $\approx 800 \mathrm{~nm}$ and length $900 \mu \mathrm{m}$ have resistivity of $1-2 \mathrm{~m} \Omega \cdot \mathrm{cm}$ along the direction of CNT alignment. The linear voltagecurrent $(I-V)$ relationship (Figure 6b) indicates that Ohmic contact is made. The conductivity of our HA-CNT ribbons is among the highest-known values measured for a CNT assembly to date (Figure 1). The measured resistivity is comparable to that of high-quality polysilicon $\left(10-20 \mathrm{Ohm} \mathrm{sq}^{-1}\right)$, which is widely used in implantable electrode arrays for cell stimulation and recording. ${ }^{[30-32]}$

Finally, the HA-CNT ribbons can be robustly transferprinted to other substrates, including flexible films of PDMS and polyimide (Kapton) as shown in Figure 6d and e. A smooth PDMS carrier substrate, made by curing and then delaminating the PDMS from a template silicon substrate, is laminated onto the original HA-CNT substrate after rolling and capillary densification. Taking advantage of kinematically controlled adhesion of PDMS to $\mathrm{SiO}_{2},{ }^{[23]}$ the CNTs stick to the PDMS when the carrier substrate is peeled quickly from the growth substrate. By laminating the PDMS carrying the HA-CNTs onto a polyimide film and then peeling it off slowly the HACNT bundles are uniformly transferred to polyimide as shown in Figure 6d and e. In contrast to previous PDMS transfer of crystallographically aligned HA-CNTs, the high packing density and uniform texture of our HA-CNT ribbons facilitates their direct printing without need to infiltrate the CNTs with a polymer as a carrier medium. ${ }^{[14]}$ Raman spectroscopy measurements again show no considerable change in the $I_{\mathrm{G}} / I_{\mathrm{D}}$ ratio, suggesting the printing process does not damage the CNTs.

To place our results in the context of previous work, it is important to review the longstanding shortfall between predicted and measured electrical properties of CNTs, and how these properties relate to the packing fraction and configuration of CNT assemblies. Such shortfalls are due to structural defects inherent in as-grown CNTs and non-uniform and non-ideal metal contacts that do not address all walls of every CNT. Further, in isotropic networks such as tangled CNT films, CNT-CNT junction resistances can far exceed the bulk resistance of CNTs over short distances. In this regard, continuous aligned CNTs are an ideal network morphology that does not suffer from such CNT-CNT resistances. Referring to Figure 1, points $(\mathrm{g})^{[16]}$ and $(\mathrm{h}),{ }^{[20]}$ which are for previous approaches to fabricating horizontally aligned CNTs show conductivities of 5 and $8 \mathrm{~m} \Omega$-cm for packing fractions of 0.45 and 0.58 , respectively.

These values are at least a factor of 5 below our results and do not facilitate tuning of the film thickness (and packing density), nor do they demonstrate transfer to flexible substrates. The conductivity of our HA-MWCNT ribbons is also comparable to SWCNT films (point $(a)^{[28]}$ ) prepared from a dispersion and then magnetically aligned using a high power source (26 Tesla). Also, the SWCNTs used in the previous study 
were made by pulsed laser ablation, which gives a CNT structural quality typically exceeding that of CVD-grown CNTs; however, the product of laser ablation is a CNT powder requiring purification to remove amorphous carbon prior to deposition and alignment. Point $(\mathrm{e})^{[29]}$ shows the highest conductivity reported for CNT assemblies to date, which is for micrometer-tall VA- MWCNTs ( $\approx 10 \%$ packing fraction), where the CNTs were grown directly on a metallic contact, and the top electrode was deposited after chemical mechanical polishing of the vertical CNTs. Thus, for best transport through CNTs, contact must be made to all the CNTs in the film as well as the inner shells of MWCNTs. Incidentally, others have reported that metal-CNT contact resistance can be reduced by thermal annealing; however, despite the non-ideal contact morphology (Figure 6c) observed for our HA-CNT devices, annealing at 200,400 , and $700{ }^{\circ} \mathrm{C}$ did not decrease the resistance.

\section{Conclusions}

We create ribbons of densely packed HA-CNTs on arbitrary substrates by combining VA-CNT growth by thermal CVD, a novel mechanical rolling process to transform the VACNTs to a horizontal orientation, and adhesion-controlled transfer printing without needing a carrier film. Control of the rolling force enables tuning of the HA-CNT ribbon thickness and packing fraction, and the ribbons can be processed by conventional lithography and etching steps to produce flexible in-plane interconnects. In spite of non-ideal contact morphology and structural defects in the CNTs, the measured electrical conductivity of our HA-CNT ribbons exceeds those of most CNT assemblies reported previously, including aligned singlewalled CNTs (SWCNTS). Future work to improve the CNT areal density, straightness, and structural quality established by the growth process and thermal annealing ${ }^{[24]}$ (e.g., at above $2000^{\circ} \mathrm{C}$ ) to heal structural defects in the CNTs may significantly increase the HA-CNT ribbon electrical conductivity. Our rolling and printing process could be scaled to full wafer areas and more complex architectures such as continuous CNT sheets and multidirectional patterns could be achieved by design of the catalyst blade pattern and/or multiple rolling and printing sequences.

\section{Experimental Section}

VA-CNT growth: A supported catalyst layer of 1-nm Fe upon $10-\mathrm{nm} \mathrm{Al}_{2} \mathrm{O}_{3}$ was deposited on (100) silicon wafers coated with 500-nm thermally grown $\mathrm{SiO}_{2}$ by sequential electron-beam evaporation of high-purity pellets of $\mathrm{Fe}$ and $\mathrm{Al}_{2} \mathrm{O}_{3}$. The catalyst was patterned by lift-off of photoresist (SPR 220) patterned by optical lithography. For lift-off, samples were sonicated for $15 \mathrm{~min}$ in acetone. CNT growth was performed in a single-zone atmospheric pressure tube furnace (Thermo-Fisher MiniMite) using a quartz tube with 22-mm inner diameter. The sample was loaded approximately $4 \mathrm{~cm}$ downstream of the control thermocouple with the long dimension of the catalyst blade patterns parallel to the gas flow. He (99.999\%), $\mathrm{H}_{2}$ (99.999\%), and $\mathrm{C}_{2} \mathrm{H}_{4}$ (99.5\%) gases were purchased from PurityPlus via Metro Welding (Detroit, MI). After flushing with $1000 \mathrm{sccm}$ He at room temperature for $10 \mathrm{~min}$, a flow of $100 / 400 \mathrm{sccm} \mathrm{He} / \mathrm{H}_{2}$ was maintained for $5 \mathrm{~min}$ to establish the catalyst annealing atmosphere before heating. The furnace was heated to the growth temperature of $775^{\circ} \mathrm{C}$ during $10 \mathrm{~min}$ and stabilized for $10 \mathrm{~min}$ under the $\mathrm{He} / \mathrm{H}_{2}$ flow, and then $100 \mathrm{sccm} \mathrm{C} \mathrm{C}_{2} \mathrm{H}_{4}$ was added for the growth duration of $20 \mathrm{~min}$. After this, the furnace was cooled rapidly by opening the clamshell enclosure while maintaining the $\mathrm{C}_{2} \mathrm{H}_{4} / \mathrm{H}_{2} / \mathrm{He}$ flow. After reaching $100^{\circ} \mathrm{C}$, the flow was switched to $\mathrm{He}$ and the sample was removed from the tube after the furnace reached $70^{\circ} \mathrm{C}$.

Microfabrication of HA-CNT ribbon interconnects: After rolling, the HA-CNTs were exposed to a brief reactive ion etch (RIE) to open their ends ( $80 \mathrm{~W}, 10 \mathrm{~Pa}, 10 \mathrm{sccm} \mathrm{O}, 30 \mathrm{~s}$ ). Next the sample was coated with $\approx 3 \mu \mathrm{m}$ of SPR220 photoresist by spining at $3000 \mathrm{rpm}$ for $30 \mathrm{~s}$ and prebaking at $115^{\circ} \mathrm{C}$ for $90 \mathrm{~s}$. The sample was exposed to UV light for $6 \mathrm{~s}$ on a Karl Suss MA- 6 Mask Aligner in order to lithographically define the shape and position of the $\mathrm{Au}$ electrodes. The sample was then post-baked at $115^{\circ} \mathrm{C}$ for $90 \mathrm{~s}$, then immersed in MIF 300 developer for 1 min. Finally $50 \AA$ Ti and $10000 \AA$ Au were electron-beam evaporated on the sample. Lift-off was performed by immersing the sample overnight in acetone and agitating after without sonication, then washing in DI water.

Characterizaton: Raman spectroscopy was performed at $\lambda=533 \mathrm{~nm}$ (Dimension P2, Lambda Solutions) through $50 \times$ magnification. SEM imaging was performed using a Philips XL30-FEG operating at $10 \mathrm{kV}$. X-ray studies were performed at the $\mathrm{G} 1$ beamline station at the Cornell High-Energy Synchrotron Source (CHESS). The wavelength of the X-rays was $0.1239 \mathrm{~nm}$ and the sample-to-detector distance was calibrated with silver behenate (first order scattering vector $q$ of $1.076 \mathrm{~nm}^{-1}$ with $q=4 \pi \sin (\theta / \lambda)$, where $2 \theta$ is the scattering angle and $\lambda$ is the wavelength). Slit collimation was used to achieve a resulting beam spot that was approximately $0.2 \mathrm{~mm}$ in height and $0.4 \mathrm{~mm}$ in width (the $y$ - and $x$-axes, respectively). A pixel array detector (PAD) system, built by H. Philipp, M. Tate, M. Hromalik, S. Gruner, and colleagues of Cornell University was used for data collection. In the GI-SAXS configuration, the sample was tilted at $0.15^{\circ}$ from the horizontal plane.

\section{Acknowledgements}

This work was funded by the University of Michigan Department of Mechanical Engineering and College of Engineering. S. Tawfick is grateful for a University of Michigan Mechanical Engineering Departmental Fellowship. We thank Eric Verploegen of Stanford University and Hugh Philipp, Arthur Woll, Mark Tate, Marianne Hromalik, and Sol Gruner of Cornell University for assistance with X-ray scattering measurements, and Brian Wardle and Enrique Garcia of MIT for valuable discussions. Microscopy and microfabrication were performed at the University of Michigan Electron Microbeam Analysis Library (EMAL) and the Lurie Nanofabrication Facility (LNF), respectively. X-ray scattering was performed at the Cornell High- 
Energy Synchrotron Source (CHESS), which is supported by the National Science Foundation and the National Institutes of Health under Grant No. DMR-0225180.

[1] R. H. Baughman, A. A. Zakhidov, W. A. de Heer, Science 2002, 297, 787.

[2] A. Loiseau, Understanding Carbon Nanotubes: From Basics to Applications, Springer, Berlin 2006.

[3] International Technology Roadmap for Semiconductors, http:// www.itrs.net/links/2007itrs/2007_chapters/2007_interconnect. pdf (accessed July 2009).

[4] H. J. Li, W. G. Lu, J. J. Li, X. D. Bai, C. Z. Gu, Phys. Rev. Lett. 2005, 95 , 086601.

[5] B. Q. Wei, R. Vajtai, P. M. Ajayan, Appl. Phys. Lett. 2001, 79, 1172.

[6] a) A. Naeemi, R. Sarvari, J. D. Meindl, IEEE Electron Device Lett. 2005, 26, 84. b) M. S. Wang, L. M. Peng, J. Y. Wang, Q. Chen, Adv. Funct. Mater. 2006, 16, 1462.

[7] S. S. Fan, M. G. Chapline, N. R. Franklin, T. W. Tombler, A. M. Cassell, H. J. Dai, Science 1999, 283, 512.

[8] D. N. Futaba, K. Hata, T. Yamada, T. Hiraoka, Y. Hayamizu, Y. Kakudate, O. Tanaike, H. Hatori, M. Yumura, S. Iijima, Nat. Mater. 2006, 5, 987.

[9] A. J. Hart, A. H. Slocum, Nano Lett. 2006, 6, 1254.

[10] B. Zhao, D. N. Futaba, S. Yasuda, M. Akoshima, T. Yamada, K. Hata, ACS Nano, 2009, 3, 108.

[11] S. J. Kang, C. Kocabas, T. Ozel, M. Shim, N. Pimparkar, M. A. Alam, S. V. Rotkin, J. A. Rogers, Nat. Nanotechnol. 2007, 2, 230.

[12] S. Huang, X. Cai, J. Liu, J. Am. Chem. Soc. 2003, 125, 5636.

[13] A. Vijayaraghavan, S. Blatt, D. Weissenberger, M. Oron-Carl, F. Hennrich, D. Gerthsen, H. Hahn, R. Krupke, Nano Lett. 2007, 7, 1556.

[14] S. J. Kang, C. Kocabas, H. S. Kim, O. Cao, M. A. Meitl, D. Y. Khang, J. A. Rogers, Nano Lett. 2007, 7, 3343.

[15] Y. Hayamizu, T. Yamada, K. Mizuno, R. C. Davis, D. N. Futaba, M. Yumura, K. Hata, Nat. Nanotechnol. 2008, 3, 289.
[16] C. Py, R. Bastien, J. Bico, B. Roman, A. Boudaoud, EPL 2007, 77, 44005 .

[17] A. J. Hart, A. H. Slocum, J. Phys. Chem. B 2006, 110, 8250

[18] C. L. Pint, Y. Q. Xu, M. Pasquali, R. H. Hauge, ACS Nano 2008, 2, 1871.

[19] D. Wang, P. C. Song, C. H. Liu, W. Wu, S. S. Fan, Nanotechnology 2008, 19, 075609.

[20] J. Shigley, T. H. Brown, Standard Handbook of Machine Design, McGraw-Hill, New York 2004.

[21] L. E. Alexander, X-ray Diffraction Methods in Polymer Science, Wiley \& Sons, New York 1969.

[22] B. N. Wang, R. D. Bennett, E. Verploegen, A. J. Hart, R. E. Cohen, J. Phys. Chem. C 2007, 111, 5859.

[23] M. A. Meitl, Z. T. Zhu, V. Kumar, K. J. Lee, X. Feng, Y. Y. Huang, I. Adesida, R. G. Nuzzo, J. A. Rogers, Nat. Mater. 2006, 5, 33.

[24] W. Huang, Y. Wang, G. H. Luo, F. Wei, Carbon 2003, 41, 2585.

[25] A. Naeemi, J. D. Meindl, presented at IEEE International Interconnect Technology Conference, Burlingame, CA, June 2-4, 2008.

[26] J. E. Fischer, W. Zhou, J. Vavro, M. C. Llaguno, C. Guthy, R. Haggenmueller, M. J. Casavant, D. E. Walters, R. E. Smalley, J. Appl. Phys. 2003, 93, 2157.

[27] K. R. Atkinson, S. C. Hawkins, C. Huynh, C. Skourtis, J. Dai, M. Zhang, S. Fang, A. A. Zakhidov, S. B. Lee, A. E. Aliev, C. D. Williams, R. H. Baughman, Phys. B 2007, 394, 339.

[28] D. Yokoyama, T. Iwasaki, K. Ishimaru, S. Sato, T. Hyakushima, M. Nihei, Y. Awano, H. Kawarada, Jpn. J. Appl. Phys. 2008, 47, 1985.

[29] G. F. Close, H. S. P. Wong, presented at Electron Devices Meeting 2007. IEDM 2007, 10-12 December 2007, Washington DC.

[30] D. J. Anderson, K. Najafi, S. J. Tanghe, D. A. Evans, K. L. Levy, J. F. Hetke, X. L. Xue, J. J. Zappia, K. D. Wise, IEEE Trans. Biomed. Eng. 1989, 36, 693.

[31] Y. Yao, A. N. Gulari, B. Casey, J. A. Wiler, K. D. Wise, 3rd International IEEE/EMBS Conference on Neural Engineering, Kohala Coast, HI, May 02-05, 2007.

[32] K. D. Wise, A. M. Sodagar, Y. Yao, M. N. Gulari, G. E. Perlin, K. Najafi, Proc.IEEE 2008, 96, 1184.

Received: May 5, 2009

Published online: August 14, 2009 